\title{
Lesions of the Tegmental Pedunculopontine Nucleus Block the Rewarding Effects and Reveal the Aversive Effects of Nicotine in the Ventral Tegmental Area
}

\author{
Steven R. Laviolette, ${ }^{1}$ Tania O. Alexson, ${ }^{2}$ and Derek van der Kooy ${ }^{1,2}$ \\ ${ }^{1}$ Neurobiology Research Group, Department of Anatomy and Cell Biology, and 2Institute of Medical Sciences, \\ University of Toronto, Toronto, Ontario, Canada M5S 1A8
}

Nicotine, the primary psychoactive component of tobacco smoke, is known to possess potent rewarding and aversive stimulus properties. The mammalian ventral tegmental area (VTA) is involved importantly in the mediation of the motivational effects of nicotine. However, the neural outputs from the VTA that may be involved in the transmission of the rewarding and aversive motivational effects of nicotine are not well understood. We report that bilateral lesions of the tegmental pedunculopontine nucleus (TPP) double dissociate the rewarding and aversive motivational effects of nicotine. Using a conditioned place preference paradigm, bilateral TPP lesions blocked a nicotine reward signal and revealed the aversive motivational properties of intra-VTA nicotine. These same TPP lesions did not block an aversive nicotine signal, as measured in a conditioned taste aversion paradigm. TPP lesions also produce an attenuation in nicotine-induced locomotor activity; however, neither learning nor performance deficits can account for these observed effects, because TPP-lesioned animals still showed clear aversive nicotine conditioning in two separate behavioral paradigms. Our results suggest that the rewarding effects of nicotine in the VTA are dependent on a nondopaminergic, descending reward pathway to the brainstem TPP.

Key words: ventral tegmental area; pedunculopontine tegmental nucleus; nicotine; reward; addiction; aversion
Nicotine, the primary psychoactive component in tobacco smoke, possesses powerful rewarding and aversive stimulus properties (Jorenby et al., 1990; Rose and Corrigall, 1997). The biphasic motivational properties of nicotine are mediated through dopaminergic and nondopaminergic neural substrates within the ventral tegmental area (VTA), and within the VTA nicotine has been demonstrated to dose-dependently produce both rewarding and aversive effects (Laviolette and van der Kooy, 2002). We have reported previously that whereas the aversive properties of both systemic and intra-VTA nicotine are dependent on mesolimbic dopamine (DA) transmission, the rewarding effects of intra-VTA nicotine are mediated through a DA-independent VTA neuronal substrate (Laviolette and van der Kooy, 2002).

The tegmental pedunculopontine nucleus (TPP) is a brainstem structure that has been identified as an important mediator of a variety of drug reward phenomena. Lesions of the TPP have been shown to block the rewarding properties of drug stimuli such as opiates (Bechara and van der Kooy, 1992; Nader and van der Kooy, 1997; Olmstead et al., 1998) as well as the rewarding properties of natural stimuli, including food and sex (Bechara and van der Kooy, 1992; Kippen and van der Kooy, 2001), in drugnaive and nondeprived states. Furthermore, excitotoxic lesions of the TPP have been reported to attenuate intravenous nicotine self-administration (Lanca et al., 2000b), suggesting that the TPP may serve to mediate the positive reinforcing properties of nicotine.

\footnotetext{
Received May 1, 2002; revised June 24, 2002; accepted July 1, 2002.

This work was supported by Canadian Institutes of Health Research.

Correspondence should be addressed to Steven R. Laviolette, Neurobiology Research Group, Department of Anatomy and Cell Biology, University of Toronto, 1 King's College Circle, Toronto, Ontario, Canada M5S 1A8. E-mail: SRLaviolette@netscape.net.

Copyright (C) 2002 Society for Neuroscience $\quad 0270-6474 / 02 / 228653-08 \$ 15.00 / 0$
}

The functional connections between the TPP and VTA have been well documented. There is a functional, predominantly cholinergic ascending projection from the TPP to the VTA (Woolf, 1991; Oakman et al., 1995; Blaha et al., 1996). These cholinergic projection terminals synapse on both GABAergic and DAergic VTA neurons (Garzon et al., 1999). In addition, there is a descending projection from the VTA to the TPP (Semba and Fibiger, 1992; Steininger et al., 1992). These descending inputs to the TPP from the VTA originate primarily from non-DA, presumably GABAergic, VTA neurons (Swanson, 1982; Goldsmith and van der Kooy, 1988).

In the present study, we performed discrete, bilateral neurotoxic lesions of the TPP and then microinfused nicotine directly into the VTA. Using an unbiased place conditioning paradigm (CPP), we examined the possible behavioral and motivational effects of these lesions on the motivational properties of nicotine in the VTA. Furthermore, we directly assessed the possible effects of TPP lesions on the aversive motivational properties of nicotine using a conditioned taste avoidance paradigm and also examined the effects of TPP lesions on nicotine-induced locomotor activity. We report that lesions of the TPP block the rewarding properties of intra-VTA nicotine and also reveal the aversive effects of intra-VTA nicotine. Interestingly, these same lesions had no effect on the aversive stimulus properties of nicotine as measured in the conditioned taste aversion (CTA) paradigm, suggesting that the TPP is essential for the selective transmission of a nicotine reward signal but not for the mediation of the aversive effects of nicotine.

\section{MATERIALS AND METHODS}

Animals and surgery. Male Wistar rats (Charles River) weighing 300-350 $\mathrm{gm}$ at the start of the experiments were anesthetized with sodium pentobarbital (Somnotol, $0.8 \mathrm{ml} / \mathrm{kg}$, i.p.) and placed in a stereotaxic 
device. Twenty-two gauge, stainless steel guide cannulas (Plastics One, Roanoke, VA) were bilaterally implanted $2 \mathrm{~mm}$ dorsal to the VTA at a $10^{\circ}$ angle using the following stereotaxic coordinates from bregma (in $\mathrm{mm}$ ): anteroposterior (AP), -5.0 ; lateral (L), \pm 2.3 ; and from the dural surface, ventral $(\mathrm{V}),-8.0$. All coordinates were taken from the atlas of Paxinos and Watson (1986). Cannulas were secured to the skull surface with jeweler's screws and dental acrylic. All experimental protocols were approved by the Institutional and Governmental Animal Care Committee guidelines.

Excitotoxic TPP lesions. Lesions of the TPP $(n=12)$ were created bilaterally by injecting NMDA ( $0.1 \mathrm{M}$; Research Biochemicals, Natick, $\mathrm{MA}$ ) in a volume of $0.25 \mu \mathrm{l}$ of physiological saline, $\mathrm{pH}$ adjusted to 7.4. Sham lesion control animals $(n=12)$ received bilateral injections of the physiological saline vehicle. Microinf usions were performed with a $1 \mu \mathrm{l}$ Hamilton (Reno, NV) microsyringe for a $20 \mathrm{~min}$ period per hemisphere. The infusion rate for NMDA was $0.02 \mu \mathrm{l} / \mathrm{min}$, after which the injector was left in place for an additional 5 min to allow diff usion of the solution from the injector tip. The injection coordinates for the TPP were the following from bregma (in $\mathrm{mm}$ ): AP, $-7.8 ; \mathrm{L}, \pm 1.6$; and from the dural surface, V, -6.6. For animals that received both intra-VTA cannulas and TPP lesions, the two surgical procedures were performed at the same time. Animals were given at least 2 weeks of recovery time before any conditioning procedures were begun.

Histological analysis. At the end of experiments, animals were deeply anesthetized with sodium pentobarbital (Somnotol, $0.8 \mathrm{ml} / \mathrm{kg}$, i.p.) and were perfused transcardially with $200 \mathrm{ml}$ of PBS $(0.1 \mathrm{M})$ followed by 400 $\mathrm{ml}$ of $4 \%$ paraformaldehyde. Brains were rapidly removed and stored for $16 \mathrm{hr}$ in a $4 \%$ paraformaldehyde postfixative. After postfixation, brains were transferred to a $25 \%$ sucrose solution in phosphate buffer $(0.1 \mathrm{M})$ for $24 \mathrm{hr}$. Brains were then flash frozen at $-70^{\circ} \mathrm{C}$, sliced in a freezing microtome into $40-\mu \mathrm{m}$-thick sections, and mounted on gelatin-coated slides. Alternate sections of the TPP were separately processed for either cresyl violet staining or for NADPH (Sigma, St. Louis, MO) histochemical staining (described below). Intra-VTA cannulae placements were verified with light microscopy using the atlas of Paxinos and Watson (1986). TPP lesions were analyzed by light microscopy with both cresyl violet and NADPH staining techniques. NADPH histochemistry was performed according to the method of Vincent et al. (1983). Mounted sections of the TPP were preincubated for $10 \mathrm{~min}$ in $0.003 \%$ Triton $\mathrm{X}-100$ in phosphate buffer $(0.1 \mathrm{M})$. Sections were then rinsed in phosphate buffer and incubated for $3.5-5 \mathrm{hr}$ in the NADPH-diaphorase staining solution, which consisted of $0.5 \mathrm{mg} / \mathrm{ml} \beta$-NADPH (Sigma) and $0.2 \mathrm{mg} / \mathrm{ml}$ nitroblue tetrazolium (Sigma) in $0.003 \%$ Triton X-100 in phosphate buffer $(0.1 \mathrm{M})$. Slides were then rinsed in $0.3 \mathrm{M}$ phosphate buffer for 3 rinses, allowed to dry, and then rinsed in graded alcohols. Slides were coverslipped with Permount. This histochemical technique selectively stains for the cholinergic cells of the reticular brainstem (Vincent et al., 1983; Laviolette et al., 2000).

Place conditioning. All animals (TPP-lesioned, $n=12$; shams, $n=12$ ) were conditioned using a standard place conditioning procedure (Nader and van der Kooy, 1997). Conditioning took place in one of two distinct environments that differed in color, texture, and smell. One environment was white with a wire mesh floor that was covered in wood chips. The other environment was black with a smooth Plexiglas floor that was wiped down with a $2 \%$ acetic acid solution before each conditioning session. The dimensions of the conditioning environments were $15 \times$ $30 \times 30$ inches $($ height $\times$ width $\times$ length). All conditioning and testing were performed in the light cycle. Animals displayed no baseline preference for either of these two conditioning environments (Laviolette et al., 2002). Animals received four drug environment alternating with four saline environment conditioning sessions, and exposure to conditioning environments (as well as whether the first trial was a drug or saline trial) was fully counterbalanced in all experiments. Each conditioning session lasted $40 \mathrm{~min}$. At testing, 1 week after the completion of conditioning (all animals were tested drug-free), animals were placed on a narrow, neutral gray zone that separated the two test compartments. Times spent in each environment were scored separately for each animal over $10 \mathrm{~min}$.

Conditioned taste aversion. For CTA experiments, subsets of both TPP-lesioned $(n=7)$ and sham-lesioned $(n=9)$ animals were randomly selected from the intra-VTA nicotine place-conditioning experiments. All CTA experiments were performed after completion of the placeconditioning experiments. Animals were trained to consume water on a limited-access regimen of $15 \mathrm{~min} / \mathrm{d}$ for $5 \mathrm{~d}$ before the commencement of conditioning. On this regimen, animals maintain $\sim 80 \%$ of their initial body weight. Training consisted of 10 exposures over a 10 -d period to an unsweetened $0.3 \%$ solution of either grape- or cherry-flavored Kool-Aid (animals display no baseline preference for either of these flavors; Jaeger and van der Kooy, 1996) for 15 min. After this, animals received a subcutaneous injection of nicotine $(0.8 \mathrm{mg} / \mathrm{kg})$. On the alternate day, the animal was exposed to the other flavor and then received a subcutaneous injection of saline. The drug-paired flavors and the day of first drug exposure were counterbalanced within groups. After conditioning, animals were left untreated for $2 \mathrm{~d}$, during which time they were given 60 min of normal water access per day. On test day, animals were presented with both the drug and saline-paired flavored solutions (animals were tested drug free) and amounts consumed of both flavors over a $20 \mathrm{~min}$ period were recorded. The presence of intra-VTA cannulas and previous intra-VTA nicotine exposure in these animals appeared to have no effect on drinking behavior, because animals were fully capable of normal fluid consumption and drank fluid amounts comparable with those of rats that did not have surgery (Stefurak and van der Kooy, 1992).

Locomotor activity. For locomotor activity experiments, randomly selected subgroups of both lesioned $(n=5)$ and sham-lesioned $(n=5)$ animals (that had been used previously in the CPP but not the CTA experiments) were used. All locomotor experiments were performed after the CPP experiments. Locomotor activity was measured in a sound- and light-attenuated test chamber that was dark gray. The dimensions of the test apparatus were $15 \times 30 \times 60$ (height $\times$ width $\times$ length) inches (dimensions identical to those used in the CPP testing apparatus). The activity of the animal was observed through the clear plastic front of the test box. One activity count constituted the animal moving its entire body over a center line separating the two halves of the apparatus. Animals were habituated to the locomotor box for a total of 120 min over 2 consecutive days before locomotor experiments. Locomotor activity was measured for $40 \mathrm{~min}$ after drug or saline injection (the same period used for place-conditioning sessions). Lesioned or sham animals received an initial injection of systemic saline to measure baseline levels of activity. After this, animals received three doses of nicotine $(0.2,0.8$, and $1.2 \mathrm{mg} / \mathrm{ml}$, s.c.) on separate days and in a totally counterbalanced order of nicotine concentrations. The observer was blind as to whether the animal was in the lesioned or sham lesion control group. Because animals had previous exposure to intra-VTA nicotine, it is possible that responses to the systemic nicotine injections were influenced by this previous VTA nicotine experience. However, the dose of intra-VTA nicotine $(24 \mathrm{nmol})$ used in the CPP experiments produces no significant locomotor-activating effects by itself (our unpublished observations) and at the beginning of systemic nicotine locomotor testing, animals had not received any intra-VTA nicotine exposure for at least $14 \mathrm{~d}$.

Drugs and injection procedures. Nicotine-di-D-tartrate (Research Biochemicals) was dissolved in physiological saline, $\mathrm{pH}$ adjusted to 7.4. Bilateral intra-VTA microinfusions of nicotine or physiological saline (control, $0.5 \mu \mathrm{l}$ volume per infusion) were performed over $1 \mathrm{~min}$. Injectors were left in place for a further $1 \mathrm{~min}$ to allow adequate diffusion from the injector tip. Animals were placed in the conditioning environments immediately after injection.

Data analysis. All data were analyzed with one- or two-way ANOVA, or Student's $t$ tests, where appropriate. Post hoc analyses were performed with Newman-Keuls or Fisher's least significant difference tests.

Preliminary intra-VTA and systemic nicotine dose selection. A previously performed dose-response analysis of intra-VTA nicotine revealed that bilateral microinfusions of nicotine dose-dependently produced potent rewarding effects, as measured in the CPP paradigm (data from Laviolette and van der Kooy, 2002) (see Fig. 4). Although a lower concentration $(0.8 \mathrm{nmol})$ of intra-VTA nicotine produced no place-conditioning effects, a higher concentration of $8 \mathrm{nmol}$ produced a conditioned place preference for the intra-VTA nicotine-paired environment. A higher concentration of intra-VTA nicotine $(24 \mathrm{nmol})$ produced the strongest place preference for the nicotine-paired environments, whereas the highest dose tested $(80 \mathrm{nmol})$ produced inconsistent conditioning and seizures in some animals, indicating a lack of behavioral specificity at this concentration. In the present study, we selected the maximally rewarding concentration of intra-VTA nicotine $(24 \mathrm{nmol})$ for testing in TPP- and sham-lesioned animals. We have reported previously that these concentrations of intra-VTA nicotine produce anatomically and pharmacologically specific effects within the VTA. Microinfusions of nicotine dorsal to the VTA or caudal to the VTA in the interpeduncular nucleus produce no motivational effects (Laviolette and van der Kooy, 2002). Nicotine has been shown to produce rapid nicotinic receptor desensitization in vitro (Dani et al., 2000). It is possible that intra-VTA microinfusions of nicotine induce some receptor desensitization. However, these 

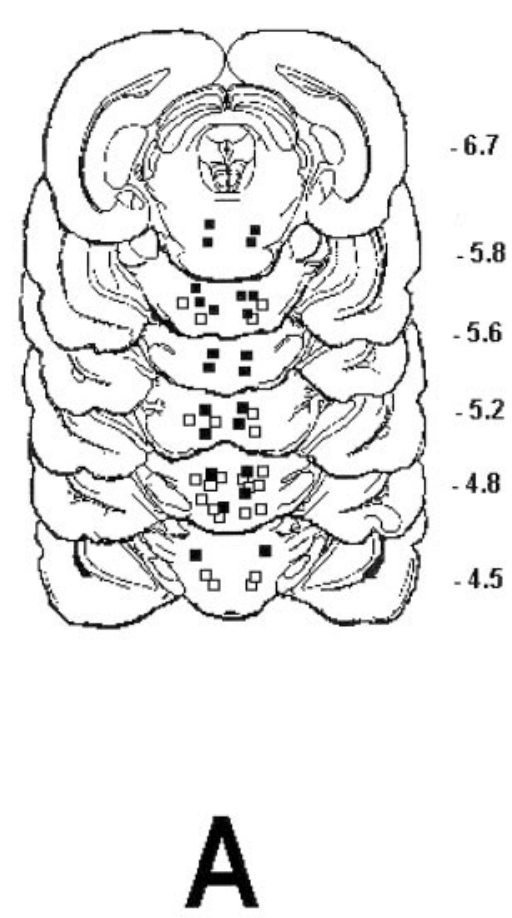

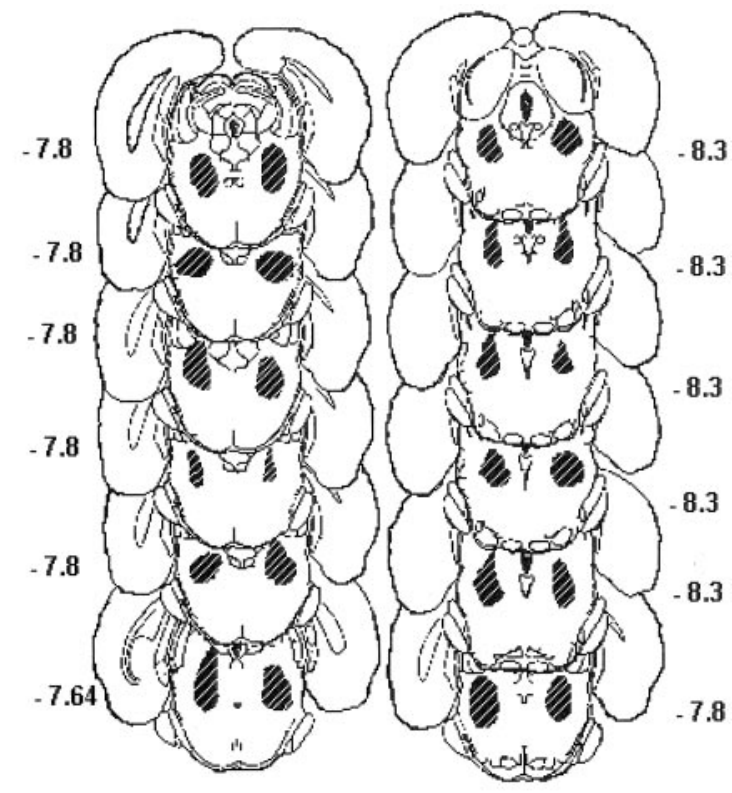

B
Figure 1. Histological analysis of bilateral intra-VTA cannula placements and bilateral excitotoxic lesions of the TPP. $A$, Schematic representation of bilateral intra-VTA cannula placements. Black squares represent cannula placements for TPP-lesioned animals $(n=12)$, and white squares represent cannula placements for sham-lesioned animals $(n=$ 12). $B$, Schematic presentation of bilateral NMDA-induced excitotoxic lesions of the TPP. Hatched areas in each section represent the maximal area of bilateral TPP damage for one individual animal $(n=12)$. effects are not likely responsible for the observed behavioral motivational effects (as measured in the CPP paradigm) in the present study, because the doses of intra-VTA nicotine used in the present study are completely blocked by behaviorally specific concentrations of the competitive nicotinic acetylcholine receptor (nAChR) antagonists di-hydro- $\beta$-erythroidine and methyllycaconitine citrate (Laviolette and van der Kooy, 2001a). For the conditioned taste aversion experiment, we selected a dose of systemic nicotine $(0.8 \mathrm{mg} / \mathrm{kg}$, s.c. $)$ that we have determined previously produces a strong aversive effect, as measured in the CPP paradigm (Laviolette and van der Kooy, 2002).

\section{RESULTS}

\section{Histological analysis}

Examination of cresyl violet-stained sections of VTA revealed that bilateral intra-VTA injector tips were localized to the anatomical boundaries of the VTA as defined by Paxinos and Watson (1986). Two animals, that were found to have injector tips outside of a $0.5 \mathrm{~mm}$ distance from the VTA, were excluded from the behavioral analyses. Correct VTA placements were located in both the rostral and caudal areas of the VTA, and no behavioral differences were apparent after microinfusions in animals that had more rostral or more caudal placements. A schematic showing intra-VTA cannula placements in the TPP and sham lesion groups is presented in Figure $1 A$.

Excitotoxic lesions of the TPP were examined with both cresyl violet staining and with NADPH-diaphorase histochemistry, which selectively stains cholinergic cell bodies within the brainstem (Vincent et al., 1983). NADPH histochemistry provides a clear, cytoarchitectonic distribution of cholinergic neurons of the TPP, whereas analysis with cresyl violet provides a measure of the extent of the lesion-induced gliotic reaction around the injection site. Figure $1 B$ shows a schematic reconstruction of the centers of the TPP lesions (indicated by hatched bars), one for each individual rat $(n=12)$. Bilateral TPP lesions varied in the magnitude of lesion-induced gliosis when analyzed with cresyl violet staining light microscopy (Fig. 1B).

Figure 2 presents microphotographs showing NADPH-stained hemisections of the TPP in both a sham-lesioned animal (Fig. 2A) and an NMDA-lesioned animal (Fig. 2B). All microphotographs are from bilaterally lesioned animals $(n=3)$; however, only one hemisphere is shown from each individual rat. Darkly stained, NADPH-positive cells are readily visible in the sham-lesioned animal. In contrast, no NADPH-positive staining is observable in the NMDA-lesioned animal. However, tissue damage resulting from the microinjector needle puncture is observable in the region of the TPP (Fig. $2 B$ ). Figure $2 C$ shows a cresyl violetstained section from a TPP-lesioned animal. The extent of the NMDA-induced lesion (as indicated by extensive reactive gliosis) is clearly present in the region of the TPP (Fig. $2 C$ ). In Figure 3, $A$ and $B$, NADPH-stained TPP sections are presented at a higher magnification. Large numbers of NADPH-positive TPP cells are present in sham-lesioned animals (Fig. $3 A$ ). In contrast, TPPlesioned animals displayed a dramatic loss of NADPH-positive cells in the TPP, although a few NADPH-positive cells are still observable within the TPP region (Fig. $3 B$ ).

\section{TPP lesions block the rewarding effects of and reveal the aversive effects of intra-VTA nicotine}

To examine the potential effects of TPP lesions on the rewarding properties of intra-VTA nicotine, we selected the maximally rewarding dose of intra-VTA nicotine (see Materials and Methods) for this series of experiments, based on a previously reported intra-VTA nicotine dose-response analysis (Fig. 4). In animals with sham lesions of the TPP $(n=12)$, intra-VTA nicotine (24 nmol) produced a robust CPP for the nicotine-paired environment when tested drug-free (Fig. 5A). Interestingly, in TPPlesioned animals, this identical dose of intra-VTA nicotine (24 nmol) produced a strong conditioned place aversion (CPA) for the nicotine-paired environment, indicating that TPP lesions had switched the motivational valence of nicotine from rewarding to aversive (Fig. 5A). An ANOVA revealed a significant interaction between groups (control vs sham vs lesioned) and treatment (intra-VTA nicotine or saline) on times spent in nicotine versus saline-paired environments $\left(F_{(2,69)}=52.3 ; p<0.05\right)$, and a 


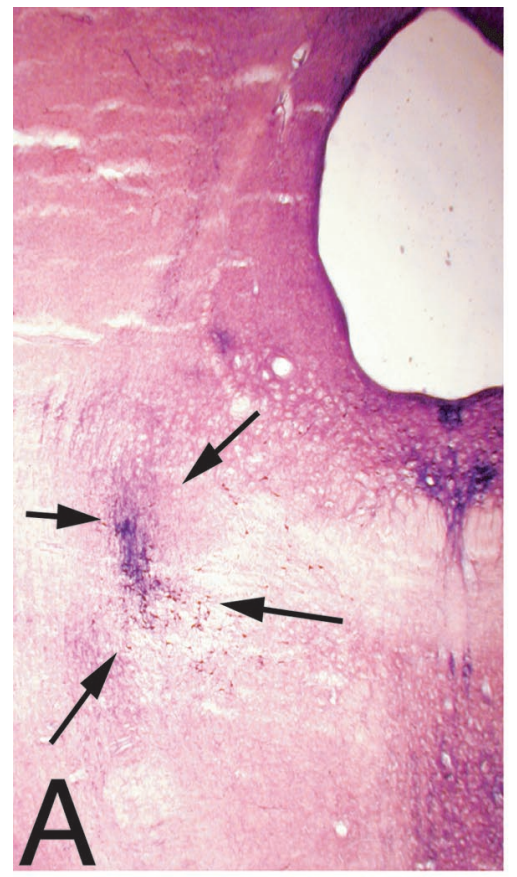

sham

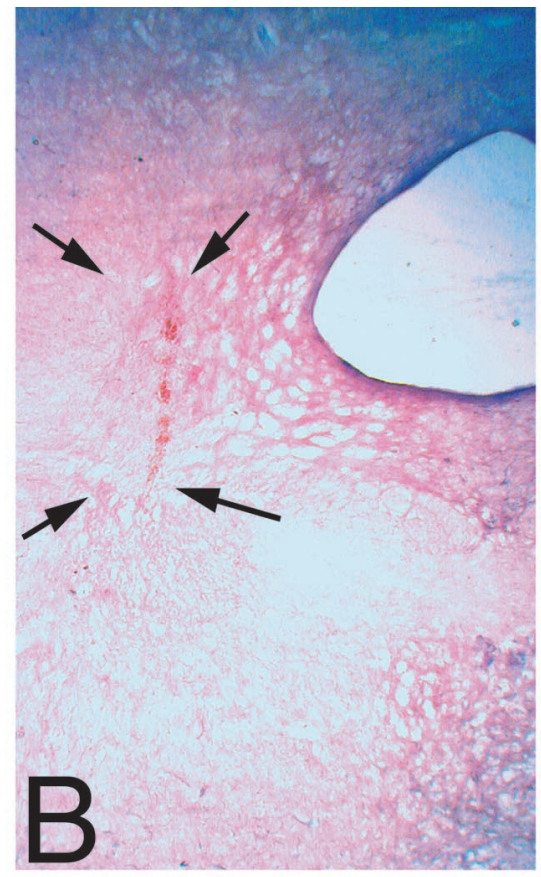

lesion

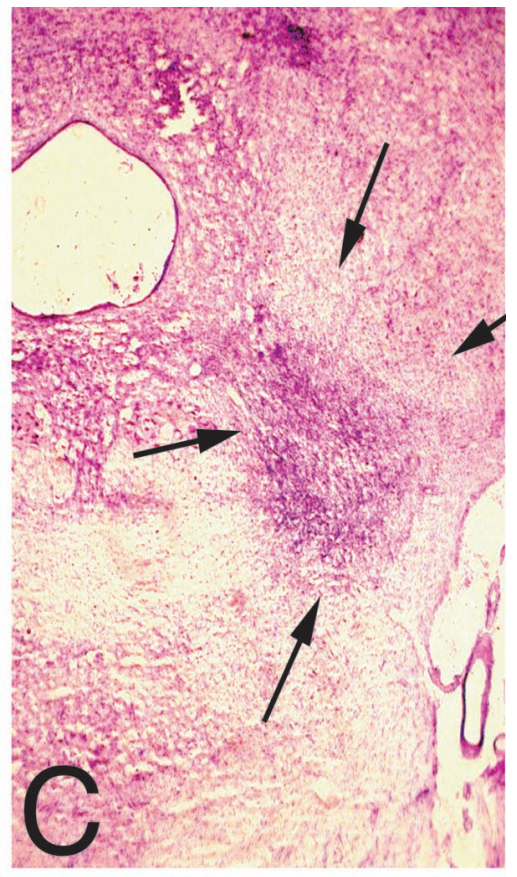

lesion

Figure 2. Histological analysis of sham or excitotoxic TPP lesions with NADPH-diaphorase histochemistry or cresyl violet staining. $A$, Coronal section (magnification, $4 \times$ ) of NADPH-stained TPP from a sham-lesioned animal. Arrows indicate approximate boundaries of the nonlesioned, intact TPP, and dark intact $\mathrm{Ch} 5$ cholinergic TPP staining is concentrated within the TPP. $B$, Coronal section (magnification, $4 \times$ ) of an NADPH-stained TPP region from a lesioned animal. Arrows indicate the approximate boundary of the TPP. Some tissue damage is present in the lesion center corresponding to the location of the injector tip. Note the absence of NADPH cholinergic staining. $C$, Coronal section (magnification, $4 \times$ ) of cresyl violet-stained TPP regions from a lesioned animal. Arrows indicate boundaries of lesion damage, as indicated by the presence of extensive gliosis in the TPP lesion area.

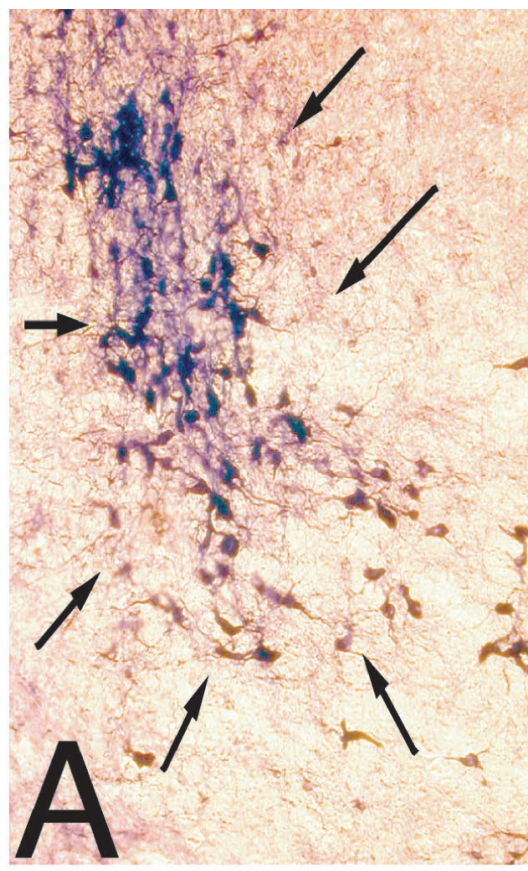

sham
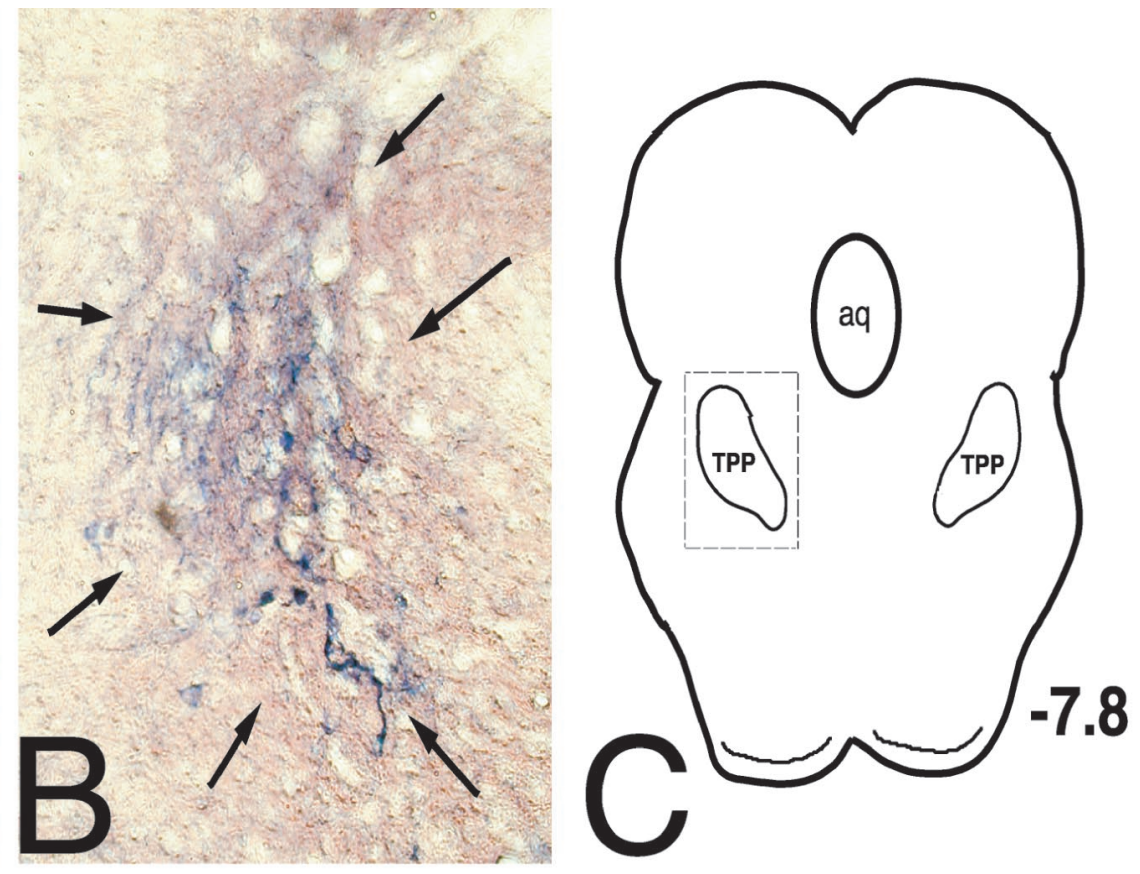

\section{lesion}

Figure 3. Histological analysis of sham and excitotoxic TPP lesions with NADPH-diaphorase histochemistry. $A$, Microphotograph (magnification, $10 \times$ ) of a coronal NADPH-stained TPP section from a sham-lesioned animal. Arrows indicate approximate neuroanatomical boundaries of the intact, nonlesioned TPP. Darkly stained cholinergic TPP cells are concentrated within the TPP region, especially in the dorsolateral TPP. $B$, Microphotograph (magnification, $10 \times$ ) of a coronal NADPH-stained TPP section from a lesioned animal. Arrows indicate approximate boundaries of the TPP. Considerable gliosis is present within the neuroanatomical boundaries of the TPP, and most NADPH-positive TPP cells have been ablated by the lesion. $C$, Schematic of a coronal section showing the approximate anatomical TPP region from which the sections displayed in $A$ and $B$ were taken. $a q$, Aqueduct. 
nicotine-paired environment saline-paired environment

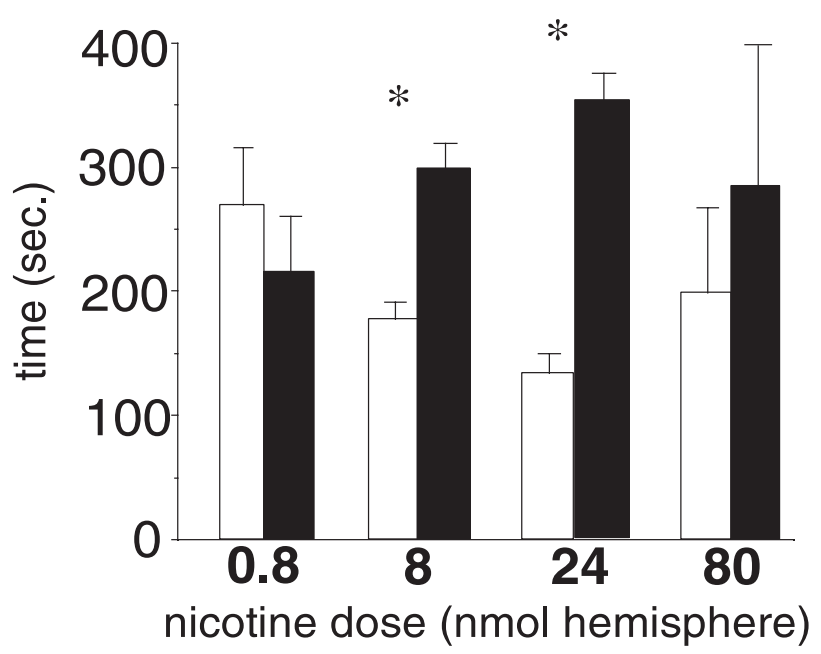

Figure 4. Motivational effects of nicotine in the ventral tegmental area. Bilateral microinfusions of nicotine ( 8 and $24 \mathrm{nmol}$ ) into the VTA produce robust rewarding effects, as demonstrated by significant place preferences for environments previously paired with these concentrations of intra-VTA nicotine $(* p<0.05)$. Data are reproduced by permission from those of Laviolette and van der Kooy (2002).

significant main effect of treatment (intra-VTA saline or nicotine) on times spent in nicotine versus saline-paired environments $\left(F_{(1,69)}=26.4 ; p<0.05\right)$. Post hoc analysis revealed that whereas both control (intra-VTA cannulas but no TPP manipulations; $n=$ $10)$ and TPP sham-lesioned animals $(n=12)$ spent significantly greater time in the nicotine-paired relative to saline-paired environments $(p<0.05)$, TPP-lesioned animals spent significantly greater time in the saline-paired relative to nicotine-paired environments $(n=12 ; p<0.05)$. On the subsequent day, we randomly selected a subgroup of animals from both the sham-lesioned $(n=$ $7)$ and TPP-lesioned animals $(n=7)$ to test in the presence of the intra-VTA nicotine cue. The purpose of this experiment was to determine whether the observed behavioral dissociation we observed when we had tested the animals without any drug cue (Fig. $5 A$ ) may have been the result of a learning deficit induced by the TPP lesions. Thus, TPP lesions may have produced a cue retrieval deficit at the time of testing and thereby prevented the expression of the intra-VTA nicotine place preference. When these groups were tested in the presence of the intra-VTA nicotine cue (24 nmol), we observed results similar to those described previously (Fig. 5B). That is, although sham-lesioned animals still displayed a significant preference for the nicotine-paired environment, TPP-lesioned animals continued to display a robust conditioned place aversion to the nicotine-paired environment (Fig. 5B). ANOVA revealed a significant interaction between groups (sham vs lesioned) and treatment (intra-VTA nicotine or saline) on times spent in nicotine-paired relative to saline-paired environments $\left(F_{(1,29}=17.4 ; p<0.05\right)$. Post hoc analyses revealed that whereas sham-lesioned animals spent significantly greater time in the nicotine-paired relative to saline-paired environments $(p<$ $0.05)$, TPP lesioned animals spent significantly greater time in the saline-paired relative to nicotine-paired environments. Thus, TPP lesions reversed the motivational valence of nicotine from rewarding to aversive. This effect was present regardless of
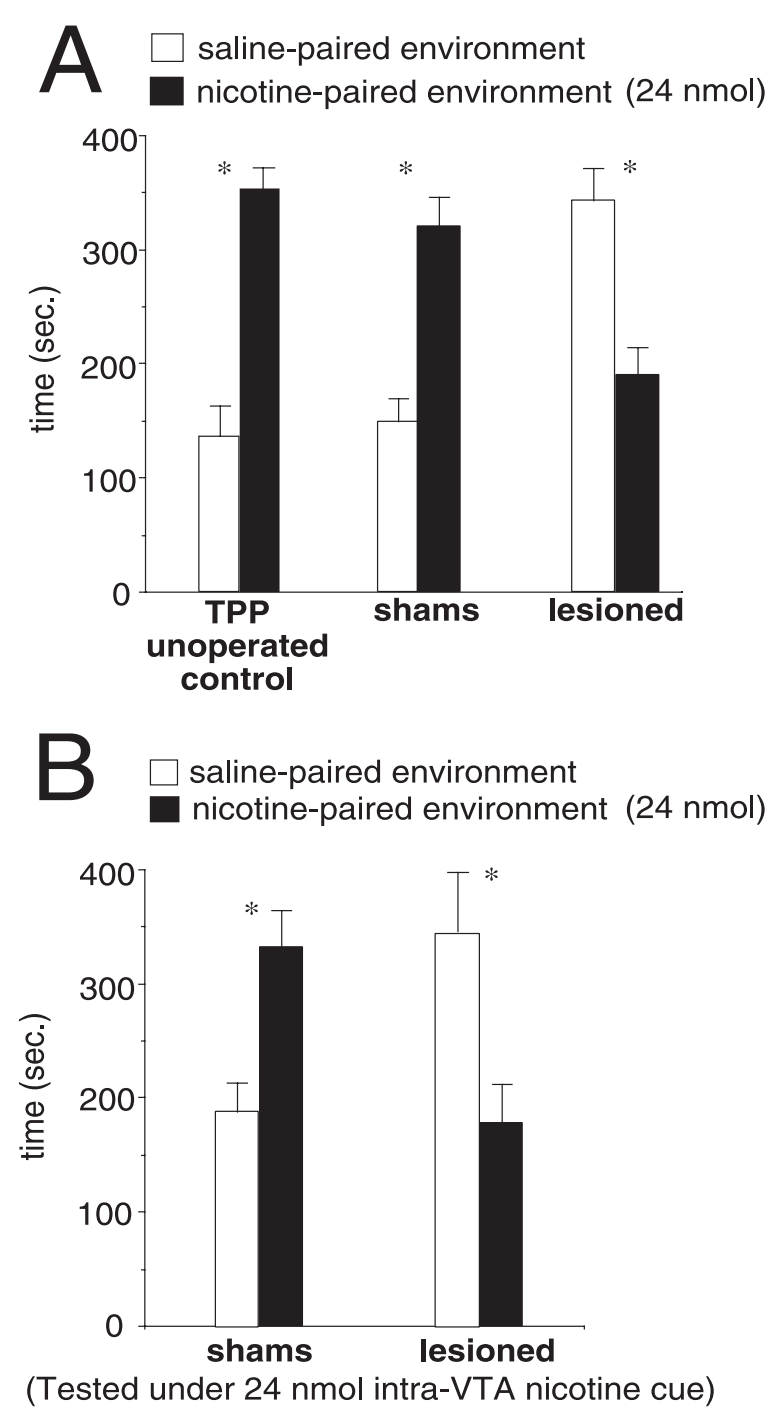

Figure 5. Motivational effects of intra-VTA nicotine in TPP controls that did not have surgery and sham- and TPP-lesioned animals tested in the presence or absence of an intra-VTA nicotine cue $(24 \mathrm{nmol})$. Error bars indicate SEM for this and subsequent figures. $A$, Animals receiving no TPP manipulations (TPP unoperated control; $n=10$ ) display a robust conditioned place preference for the environment paired with nicotine $(* p<0.05)$. Similarly, animals with sham lesions of the TPP $(n=12)$ displayed a robust conditioned place preference for the environment paired with intra-VTA nicotine $(* p<0.05)$. In contrast, animals with excitotoxic bilateral TPP lesions $(n=12)$ demonstrated a reversal in the motivational valence of intra-VTA nicotine and displayed a significant aversion to the environment paired with intra-VTA nicotine $\left({ }^{*} p<0.05\right)$. $B$, Both sham- and TPP-lesioned animals were retested in the presence of the intra-VTA nicotine cue. Although sham-lesioned animals continued to display a robust conditioned place preference for the environment paired with intra-VTA nicotine $\left({ }^{*} p<0.05\right)$, TPP-lesioned animals continued to demonstrate a significant aversion to the environment paired with intra-VTA nicotine $(* p<0.05)$.

whether the intra-VTA nicotine cue was present or absent at the time of testing.

\section{TPP lesions do not block the aversive stimulus properties of nicotine}

In addition to producing rewarding effects, nicotine also possesses strong aversive stimulus properties (Jorenby et al., 1990). We examined whether TPP lesions would affect the aversive proper- 


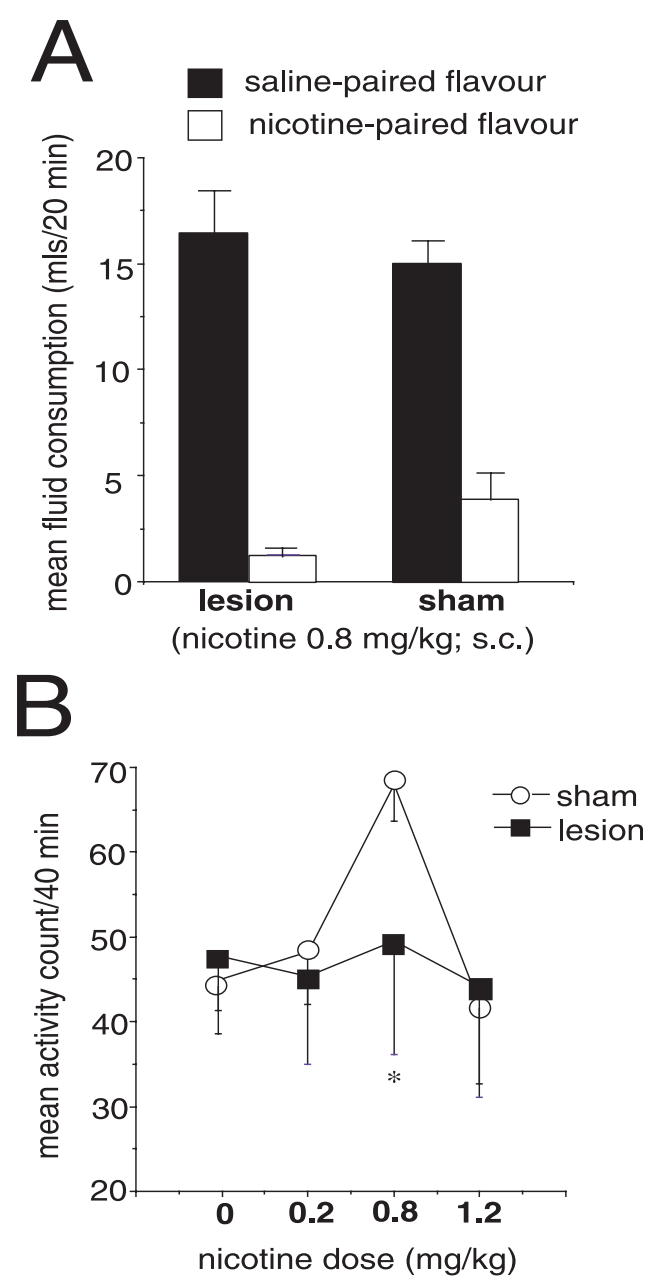

Figure 6. $A$, Systemic nicotine $(0.8 \mathrm{mg} / \mathrm{kg}$, s.c.) and conditioned taste aversions in sham- versus TPP-lesioned animals. Left, Both TPP-lesioned $(n=7)$ and sham-lesioned $(n=9)$ animals displayed robust conditioned taste aversions to flavors previously paired with the nicotine cue $(0.8$ $\mathrm{mg} / \mathrm{kg}$, s.c.; $\left.{ }^{*} p<0.05\right)$. $B$, Systemic nicotine-induced locomotor activity in sham versus TPP lesioned animals. Data points represent mean activity counts over $40 \mathrm{~min} \pm \mathrm{SEM}$. Sham-lesioned animals $(n=5)$ displayed a significant activity count increase over baseline levels (saline, 0 dose) at a subcutaneous nicotine dose of $0.8 \mathrm{mg} / \mathrm{kg}\left({ }^{*} p<0.05\right)$. In contrast, TPPlesioned animals $(n=5)$ displayed no activity count increases above saline baseline levels at any of the tested nicotine doses.

ties of nicotine. We used a CTA paradigm to selectively sample the aversive properties of nicotine. In the CTA paradigm, animals will readily acquire aversions to flavors paired with specific drug stimuli. We selected a dose of systemic nicotine $(0.8 \mathrm{mg} / \mathrm{kg}$, s.c. $)$ for CTA experiments that we have determined previously to produce strong aversive effects, as measured in the CPP and CTA paradigms (Jorenby et al., 1990; Laviolette and van der Kooy, 2002). When animals were given a two-bottle choice test at the conclusion of CTA conditioning, both TPP-lesioned animals $(n=$ $7)$ and sham-lesioned animals $(n=9)$ displayed strong aversions to the nicotine-paired flavors, indicating that TPP lesions do not block the aversive stimulus properties of this dose of nicotine (0.8 $\mathrm{mg} / \mathrm{kg}$, s.c.) (Fig. 6A). An ANOVA revealed a significant main effect of treatment (nicotine-paired or saline-paired flavors) on absolute amounts (in milliliters) of the two fluids consumed $\left(F_{(1,31)}=63.8 ; p<0.05\right)$. However, no significant effect of group (sham vs lesioned) was observed on flavor consumption $\left(F_{(1,31}=\right.$
$0.4 ; p>0.05)$, nor was there any significant interaction of the two factors. Post hoc analyses revealed that both sham- and TPPlesioned animals consumed significantly less of the nicotinepaired flavor relative to the saline-paired flavor at testing $(p<$ 0.05). Furthermore, TPP lesions had no effect on the animals' capacity to drink, because total mean fluid consumption across conditioning trials was nearly identical between the TPP-lesioned group (mean $\pm \mathrm{SEM}, 18.6 \pm 0.4 \mathrm{ml}$ ) and sham-lesioned group (18.0 $\pm 0.8 \mathrm{ml}$; data not shown).

\section{TPP lesions attenuate nicotine-induced locomotor activity}

We selected three doses of systemic nicotine for locomotor activity experiments $(0.2,0.8$, and $1.2 \mathrm{mg} / \mathrm{kg}$, s.c.). Doses higher than $1.2 \mathrm{mg}$ rendered animals (both sham and lesioned) cataleptic and thus were not used for further testing. In sham-lesioned animals, locomotor activity was significantly greater than saline baseline levels at only one nicotine dose $(0.8 \mathrm{mg} / \mathrm{kg})$ (Fig. $6 \mathrm{~B})$. In contrast, TPP-lesioned animals displayed no significant increase in locomotor activity at any of the nicotine doses. An ANOVA revealed a significant main effect of dose on locomotor activity $\left(F_{(3,39)}=\right.$ 458.7; $p<0.05)$. Post hoc analysis revealed that locomotor activity was significantly increased in sham-lesioned animals at the 0.8 $\mathrm{mg} / \mathrm{kg}$ nicotine dose relative to saline. However, in TPP-lesioned animals, this same dose of nicotine did not increase locomotor activity levels above saline baseline levels $(p>0.05)$ (Fig. 6B), indicating that TPP lesions block the locomotor-stimulating effects of systemic nicotine.

\section{DISCUSSION}

The present results implicate the TPP as an important brainstem nucleus output for the transmission of nicotine reward signaling in the VTA. Indeed, TPP lesions not only blocked the rewarding effects of intra-VTA nicotine but also were sufficient to reveal the aversive properties of intra-VTA nicotine. This pattern of results was present regardless of whether the intra-VTA nicotine cue was present or absent at the time of testing, indicating that TPP lesions were not inducing any state-dependent learning impairments. Interestingly, lesions of the TPP failed to attenuate the aversive stimulus properties of nicotine, suggesting that the TPP is essential for the selective mediation of a nicotine reward signal. Furthermore, animals were fully capable of associating interoceptive nicotine drug cues with specific flavor stimuli, indicating that TPP lesions were not inducing any nonspecific sensory or learning impairments.

Our results are congruent with a considerable body of evidence implicating the TPP in the mediation of the rewarding effects of various stimuli, including opiates (Nader and van der Kooy, 1997; Olmstead et al., 1998), amphetamine (Bechara and van der Kooy, 1992; Olmstead and Franklin, 1994), food (Bechara and van der Kooy, 1992), saccharin (Stefurak and van der Kooy, 1994) and sex-related stimuli (Kippen and van der Kooy, 2001). To our knowledge, this is the first report of TPP lesions producing a reversal in the motivational valence of a drug stimulus.

A previous study has reported that TPP lesions attenuate intravenous nicotine self-administration (Lanca et al., 2000b). Our results are consistent with this report and would suggest that the observed decrease in nicotine self-administration observed in this study may be attributable to the ability of TPP lesions to switch the motivational valence of the nicotine cue from rewarding to aversive. The TPP substrates responsible for mediating a nicotine reward signal are unknown. Ascending cholinergic in- 
puts from both the TPP and adjacent laterodorsal tegmental nucleus (LDT) have been shown to regulate the activity of the mesolimbic DA system, suggesting that reward signaling through the mesolimbic DA system may be dependent on this cholinergic, brainstem input (Blaha and Winn, 1993; Blaha et al., 1996; Mathur et al., 1997; Laviolette et al., 2000). However, the majority of these ascending inputs to the VTA DA system arise from the LDT rather than the TPP (Oakman et al., 1995). Thus, ascending cholinergic input from the LDT rather than the TPP may be critical for the mediation of DA-dependent behavioral phenomena in the VTA (Blaha et al., 1996; Laviolette et al., 2000; Forster et al., 2002). Furthermore, functional cholinergic regulation of the VTA dopaminergic neurons appears to be dependent on muscarinic M5 subtype receptors rather than nAChRs (Yeomans et al., 2000; Forster et al., 2002), suggesting that cholinergic reward signaling via these ascending projections to the VTA are mediated through a muscarinic rather than a nicotinic mechanism.

The VTA sends descending, non-DA projections to the TPP (Swanson, 1982; Goldsmith and van der Kooy, 1988). Furthermore, it has been reported that nicotine selectively activates noncholinergic, GABAergic TPP neurons rather than cholinergic cell bodies (Lanca et al., 2000a). Approximately $30 \%$ of the neuronal population within the TPP has been identified as GABAergic (Kosaka et al., 1988; Ford et al., 1995). Corrigall et al. (2001) reported that intra-TPP microinf usion of both a $\mathrm{GABA}_{\mathrm{A}}$ receptor agonist and $\mathrm{GABA}_{\mathrm{B}}$ receptor agonist attenuated nicotine selfadministration on a fixed ratio self-administration schedule but did not influence nicotine self-administration when animals were tested in a progressive ratio self-administration paradigm (Corrigall et al., 2001). Nevertheless, because descending GABAergic inputs to the TPP arise from the GABAergic neurons of the VTA and mediate a non-DA reward signal (Laviolette and van der Kooy, 2001b), one possibility is that a non-DA, nicotine reward signal is mediated by a GABAergic receptor substrate in the TPP.

We propose that the rewarding effects of nicotine may be mediated via this descending, non-DA pathway. In this case, TPP lesions would selectively block the rewarding properties of nicotine in the VTA by removing the reward output substrate located in the TPP. In contrast, an aversive, DAergic nicotine signal in the VTA would remain intact. Indeed, DA receptor blockade completely blocks the development of nicotine CTAs and CPAs (Laviolette and van der Kooy, 2002). Together, these results double dissociate the neural substrates mediating the rewarding and aversive motivational properties of nicotine. We propose that descending VTA outputs to the TPP specifically mediate a nicotine reward signal, independently of an aversive, DA-dependent nicotine signal ascending from the VTA.

One possibility is that nicotine reward signals are mediated by GABAergic neurons in the VTA. Indeed, activation of VTA GABAergic neurons produces potent, DA-independent rewarding effects (Laviolette and van der Kooy, 2001b). Both DA and GABAergic VTA neurons possess nAChRs, and both populations are activated by nicotine (Erhardt et al., 2002; Mansvelder et al., 2002), suggesting that separate VTA substrates can mediate the motivational effects of nicotine. Nicotine produces a transient activation of VTA GABAergic neurons followed by a longerlasting activation of mesolimbic DA transmission attributable to the desensitization of nAChRs on the GABA neurons and the resulting decreased inhibition of the DA system (Erhardt et al., 2002; Mansvelder et al., 2002). Although it is difficult to compare the in vitro effects of nicotine with in vivo intra-VTA microinfusions, it is possible that the short-lasting activation of the
GABAergic VTA neurons are responsible for the acute rewarding effects of nicotine, whereas the slower and longer-lasting activation of the mesolimbic DA pathway represents a separate motivational process that mediates the aversive properties of the nicotine stimulus (Laviolette and van der Kooy, 2002). Sustained dysregulation of mesolimbic DA activity induced by nicotine may represent the dysphoric withdrawal symptoms of nicotine use. In the case of smoking behavior, repetitive intake of nicotine at regular intervals might produce immediate rewarding effects by activating non-DA VTA GABAergic neurons. However, after continued nicotine exposure and sustained activation of an aversive DA signal, nicotine may be able to alleviate the aversive motivational withdrawal effects of nicotine by restoring inhibitory balance to the mesolimbic DA system through the acute activation of the GABAergic neurons.

We observed an attenuation of systemic nicotine-induced locomotor activity in TPP-lesioned animals. As discussed previously, ascending cholinergic inputs from the TPP to the VTA have been shown to functionally influence the activity of the mesolimbic DA system (Blaha et al., 1996). Furthermore, systemic nicotineinduced locomotor activity appears to be dependent on the mesolimbic DA system (Clarke et al., 1988). Thus, one possibility is that TPP lesions produced a partial blockade of systemic nicotineinduced locomotor activity by blocking excitatory cholinergic inputs from the TPP to the VTA DA neurons. Alternatively, descending VTA inputs to the TPP may in part be responsible for the locomotor activating effects of systemic nicotine.

Nicotine has been reported to produce strong anxiogenic effects (Zarrindast et al., 2000; Irvine et al., 2001). Furthermore, lesions of the TPP have been reported to increase measures of anxiety in various behavioral assays (Leri and Franklin; 1998; Podhorna and Franklin; 1998). It has been reported that administration of anxiolytic drugs before place preference testing was able to restore morphine but not amphetamine CPPs (Leri and Franklin; 2000), suggesting that rather than blocking a specific motivational process, TPP lesions induce high levels of anxiety that simply overshadow an unblocked morphine reward signal at the time of behavioral testing. Based on this suggestion, an alternative explanation for the present findings is that TPP lesions may amplify an aversive or anxiogenic nicotine signal within the VTA, thereby overshadowing the rewarding properties of nicotine rather than interfering with a specific neural motivational process. However, this interpretation is an unlikely explanation for the present findings, because we observed not only a complete blockade of an intra-VTA nicotine reward signal but also a reversal of the motivational valence of intra-VTA nicotine from rewarding to aversive. Thus, any putative anxiogenic effects induced by TPP lesions did not overshadow the conditioning or expression of nicotine aversions. This nicotine-induced conditioned place aversion was apparent in the presence or absence of the intra-VTA nicotine cue, indicating that TPP lesions were not producing any state-dependent learning deficits. Furthermore, lesioned animals were fully capable of discriminating flavor associations with a systemic nicotine versus saline cue, again demonstrating that these animals were fully capable of associating the interoceptive nicotine stimulus (either intra-VTA or systemically) with exteroceptive cues (either environmental properties or specific flavors).

The present study is the first demonstration of a functional link between intra-VTA nicotine neural motivational substrates with the brainstem TPP. Our results suggest that the TPP is specifically required for intra-VTA nicotine reward signaling. Interest- 
ingly, TPP lesions did not attenuate the development of a conditioned taste aversion for nicotine, suggesting that the TPP is not directly involved in the mediation of the aversive stimulus properties of nicotine. Together with previous findings, our results double dissociate intra-VTA substrates that mediate the aversive and rewarding properties of nicotine. Although the rewarding effects of nicotine in the VTA are mediated by a non-DA, descending TPP-dependent neural pathway, the aversive stimulus properties of nicotine are mediated through a separate, ascending dopaminergic neural system.

\section{REFERENCES}

Bechara A, van der Kooy D (1992) A single brain stem substrate mediates the motivational effects of both opiates and food in nondeprived rats but not in deprived rats. Behav Neurosci 106:351-363.

Blaha CD, Winn P (1993) Modulation of dopamine efflux in the striatum following cholinergic stimulation of the substantia nigra in intact and pedunculopontine tegmental nucleus-lesioned animals. J Neurosci 13:1035-1044.

Blaha CD, Allen LF, Inglis WI, Das S, Latimer MP, Vincent SR, Winn $P$ (1996) Modulation of dopamine efflux in the nucleus accumbens after cholinergic stimulation of the ventral tegmental area in intact, pedunculopontine tegmental nucleus-lesioned, and laterodorsal tegmental nucleus-lesioned rats. J Neurosci 16:714-722.

Clarke PBS, Fu DS, Jakubovic A, Fibiger HC (1988) Evidence that mesolimbic dopaminergic activation underlies the locomotor stimulant action of nicotine in rats. J Pharmacol Exp Ther 246:701-708.

Corrigall WA, Coen KM, Zhang J, Adamson KL (2001) GABA mechanisms in the pedunculopontine tegmental nucleus influence particular aspects of nicotine self-administration in the rat. Psychopharmacology 158:190-197.

Dani JA, Radcliffe KA, Pidoplichko VI (2000) Variations in desensitization of nicotinic acetylcholine receptors from hippocampus and midbrain dopamine areas. Eur J Pharmacol 393:31-38.

Erhardt S, Scwieler L, Engberg G (2002) Excitatory and inhibitory responses of dopamine neurons in the ventral tegmental area to nicotine. Synapse 43:227-237.

Ford B, Holmes CJ, Mainville L, Jones BE (1995) GABAergic neurons in the rat pontomesencephalic tegmentum: codistribution with cholinergic and other tegmental neurons projecting to the posterior lateral hypothalamus. J Comp Neurol 363:177-196.

Forster GL, Yeomans JS, Takeuchi J, Blaha CD (2002) M5 muscarinic receptors are required for prolonged accumbal dopamine release after electrical stimulation of the pons in mice. J Neurosci 22:RC190:1-6.

Garzon M, Vaughn RA, Uhl GR, Kuhar MJ, Pickel VM (1999) Cholinergic axon terminals of the ventral tegmental area target a subpopulation of neurons expressing low levels of the dopamine transporter. J Comp Neurol 410:197-210.

Goldsmith M, van der Kooy D (1988) Separate non-cholinergic descending projections and cholinergic ascending projections from the nucleus tegmenti pedunculopontinus. Brain Res 445:386-391.

Irvine EE, Cheeta S, File SE (2001) Tolerance to nicotine's effects in the elevated plus maze and increased anxiety during withdrawal. Pharmacol Biochem Behav 68:319-325.

Jaeger TV, van der Kooy D (1996) Separate neural substrates mediate the motivating and discriminative properties of morphine. Behav Neurosci 110:181-201.

Jorenby DE, Steinpreis RE, Sherman JE, Baker TB (1990) Aversion instead of preference learning indicated by nicotine place conditioning in rats. Psychopharmacology 101:533-538.

Kippen TE, van der Kooy D (2001) Blockade of sexually-rewarded conditioned place preference by tegmental pedunculopontine nucleus lesions. Behav Pharmacol 12 [Suppl 1]:S52.

Kosaka TM, Tauchi M, Dahl JL (1988) Cholinergic neurons containing GABA-like and/or glutamic acid decarboxylase-like immunoreactivities in various brain regions of the rat. Exp Brain Res 70:605-617.

Lanca JA, Sanelli TR, Corrigall WA (2000a) Nicotine-induced fos expression in the pedunculopontine mesencephalic tegmentum in the rat. Neuropharmacology 13:2808-2817.

Lanca AJ, Adamson KL, Coen KM, Chow BLC, Corrigall WA (2000b) The pedunculopontine tegmental nucleus and the role of cholinergic neurons in nicotine self-administration in the rat: a correlative neuroanatomical and behavioral study. Neuroscience 96:735-742.

Laviolette SR, van der Kooy D (2001a) Blockade of the $\alpha 7$ nicotinic receptor subunit switches the motivational valence of nicotine from rewarding to aversive in the ventral tegmental area. Behav Pharmacol 12 [Suppl 1]:S57.

Laviolette SR, van der Kooy D (2001b) GABA receptors in the ventral tegmental area control bidirectional reward signalling between dopaminergic and non-dopaminergic neural motivational systems. Eur J Neurosci 13:1009-1015.

Laviolette SR, van der Kooy D (2002) Blockade of mesolimbic dopamine transmission dramatically potentiates nicotine reward sensitivity in the ventral tegmental area. Mol Psychiatry, in press.

Laviolette SR, Priebe RPM, Yeomans JS (2000) Role of the laterodorsal tegmental nucleus in scopolamine and amphetamine-induced locomotion and stereotypy. Pharmacol Biochem Behav 65:163-174.

Laviolette SR, Nader K, van der Kooy D (2002) Motivational state determines the functional role of the mesolimbic dopamine system in the mediation of opiate reward processes. Behav Brain Res 129:17-29.

Leri F, Franklin KBJ (1998) Learning impairments caused by lesions to the pedunculopontine tegmental nucleus: an artifact of anxiety? Brain Res 807:187-192.

Leri F, Franklin KBJ (2000) Diazepam modifies the effect of pedunculopontine lesions on morphine but not on amphetamine conditioned place preference. Behav Brain Res 117:21-27.

Mansvelder HD, Keath JR, McGehee DS (2002) Synaptic mechanisms underlie nicotine-induced excitability of brain reward areas. Neuron 33:905-919.

Mathur A, Shandarin A, Laviolette SR, Parker J, Yeomans JS (1997) Locomotion and stereotypy induced by scopolamine: contributions of muscarinic receptors near the pedunculopontine tegmental nucleus. Brain Res 775:144-155.

Nader K, van der Kooy D (1997) Deprivation state switches the neurobiological substrates mediating opiate reward in the ventral tegmental area. J Neurosci 17:383-390.

Oakman SA, Faris PL, Kerr PE, Cozzari C, Hartman BK (1995) Distribution of pontomesencephalic cholinergic neurons projecting to substantia nigra differs significantly from those projecting to the ventral tegmental area. J Neurosci 15:5859-5869.

Olmstead MC, Franklin KBJ (1994) Lesions of the pedunculopontine tegmental nucleus block drug-induced reinforcement but not amphetamine-induced locomotion. Brain Res 638:29-35.

Olmstead MC, Munn EM, Franklin KB, Wise RA (1998) Effects of pedunculopontine tegmental nucleus lesions on responding for intravenous heroin under different schedules of reinforcement. J Neurosc 18:5035-5044.

Paxinos G, Watson C (1986) The rat brain in stereotaxic coordinates, Ed 2. New York: Academic.

Podhorna J, Franklin KBJ (1998) Lesions of the pedunculopontine tegmental nucleus increase anxiety in rats. NeuroReport 9:1783-1786.

Rose JE, Corrigall WA (1997) Nicotine self-administration in animals and humans: similarities and differences. Psychopharmacology 130: $28-40$.

Semba K, Fibiger HC (1992) Afferent connections of the laterodorsal and pedunculopontine tegmental nuclei in the rat: a retro- and anterograde transport and immunohistochemical study. J Comp Neurol 323:387-410.

Stefurak TL, van der Kooy D (1992) Saccharin's rewarding, conditioned reinforcing, and memory-improving properties: mediation by isomorphic or independent processes? Behav Neurosci 106:125-139.

Stefurak TL, van der Kooy D (1994) Tegmental pedunculopontine lesions in rats decrease saccharin's rewarding effects but not its memory improving effect. Behav Neurosci 108:972-980.

Steininger TL, Rye DB, Wainer BH (1992) Afferent projections to the cholinergic pedunculopontine tegmental nucleus and adjacent midbrain extrapyramidal area in the albino rat. 1. Retrograde tracing studies. J Comp Neurol 321:515-543.

Swanson LW (1982) The projections of the ventral tegmental area and adjacent regions: a combined fluorescent and retrograde tracer and immunofluorescence study in the rat. Brain Res Bull 9:321-353.

Vincent SR, Satoh K, Armstrong DM, Fibiger HC (1983) NADPHdiaphorase: a selective histochemical marker for the cholinergic neurons of the pontine reticular formation. Neurosci Lett 43:31-36.

Woolf NJ (1991) Cholinergic systems in mammalian brain and spinal cord. Prog Neurobiol 37:475-524.

Yeomans JS, Takeuchi J, Baptista M, Flynn DD, Lepik K, Nobrega J, Fulton J, Ralph MR (2000) Brain-stimulation reward thresholds raised by an antisense oligonucleotide for the M5 muscarinic receptor infused near dopamine cells. J Neurosci 20:8861-8867.

Zarrindast MR, Homayoun H, Babaie A, Etminani A, Gharib B (2000) Involvement of adrenergic and cholinergic systems in nicotine-induced anxiogenesis in mice. Eur J Pharmacol 407:145-158. 